\title{
Treponema amylovorum sp. nov., a Saccharolytic Spirochete of Medium Size Isolated from an Advanced Human Periodontal Lesion $\dagger$
}

\author{
C. WYSS,${ }^{1 *}$ B. K. CHOI,${ }^{2}$ P. SCHÜPBACH, ${ }^{1}$ B. GUGGENHEIM, ${ }^{1}$ AND U. B. GÖBEL ${ }^{2}$ \\ Institut für Orale Mikrobiologie und Allgemeine Immunologie, Zentrum für Zahn-, Mund-und Kieferheilkunde der \\ Universität Zürich, CH-8028 Zürich, Switzerland, ${ }^{1}$ and Universitätsklinikum Charité, Institut für Mikrobiologie \\ und Hygiene, D-10117 Berlin, Germany ${ }^{2}$
}

\begin{abstract}
A highly motile, medium-size, saccharolytic spirochete was isolated from an advanced human periodontal lesion in medium OMIZ-Pat supplemented with $1 \%$ human serum. The growth of this organism is dependent on either glucose, maltose, starch, or glycogen. The cells contain six endoflagella, three per pole, which overlap in the central region of the cell body. On the basis of its cell morphology and enzyme activities, as well as its sodium dodecyl sulfate-polyacrylamide gel electrophoresis protein and antigen profiles, this organism is clearly distinct from all previously cultured spirochetes. The presence of a novel species is supported by the 16S rRNA sequence of this organism, which places it in phylotype 19 of Choi et al. (B. K. Choi, B. J. Paster, F. E. Dewhirst, and U. B. Göbel, Infect. Immun. 62:1889-1895, 1994). The only isolate, strain HA2P, is designated the type strain of a novel species, for which we propose the name Treponema amylovorum.
\end{abstract}

The microbial populations of dental plaque have been intensely investigated because of their implication in the development of periodontal diseases (2). Oral spirochetes often predominate in subgingival plaque at diseased sites, but little is known about the population structure of these bacteria and their biological activities. This is mainly due to difficulties encountered in the in vitro cultivation of these fastidious organisms. Recent improvements in culture media and the application of limit dilution techniques have allowed the routine isolation of oral spirochetes (7). Here, we describe a novel oral Treponema species isolated from an advanced periodontal lesion, for which we propose the name Treponema amylovorum sp. nov.; the only isolate, HA2P, is the type strain of this species.

\section{MATERIALS AND METHODS}

Bacteria. The following type and reference strains were maintained as described previously $(5,7)$ : Treponema denticola CD-1, 51B2, ATCC 33521, ATCC 35404 , and ATCC $35405^{\mathrm{T}}$; Treponema maltophilum ATCC $51939^{\mathrm{T}}$, ATCC 51940 and ATCC 51941; Treponema pectinovorum ATCC $33768^{\mathrm{T}}$; Treponema socranskii subsp. buccale ATCC $35534^{\mathrm{T}}$; Treponema socranskii subsp. paredis ATCC $35535^{\mathrm{T}}$; Treponema socranskii subsp. socranskii ATCC $35536^{\mathrm{T}}$; and Treponema vincentii LA-1 ( = ATCC 35580) and Ritz A.

Culture media. Medium OMIZ-Pat (7) supplemented with $1 \%$ heat-inactivated human serum (catalog no. H1388; Sigma) $100 \mathrm{mg}$ of fosfomycin per liter, and $1 \mathrm{mg}$ of rifampin per liter was used during the isolation of strain $\mathrm{HA} 2 \mathrm{P}^{\mathrm{T}}$ by limit dilution in 96-well microtiter plates (7). When cultures were supplemented with serum, medium OMIZ-Pat was prepared without asialofetuin. Human serum was heat treated at $56^{\circ} \mathrm{C}$ for $30 \mathrm{~min}$, clarified by centrifugation and filtration through 0.2- $\mu \mathrm{m}$-pore-size filters (type Supor 200; Gelman), and stored at $-20^{\circ} \mathrm{C}$ Solid-agarose cloning and further propagation were done in the absence of antibiotics as described previously (7).

Phenotypic characterization. Determinations of enzyme activities and the growth-promoting effects of medium components, as well as other analyses, such as sodium dodecyl sulfate-polyacrylamide gel electrophoresis (SDS-PAGE), Western blotting, and electron microscopy, were performed as previously described (7).

${ }^{*}$ Corresponding author. Mailing address: Institut für Orale Mikrobiologie und Allgemeine Immunologie, Plattenstr. 11, Postfach, $\mathrm{CH}-$ 8028 Zürich, Switzerland. Phone: 01 2573322. Fax: 01 2615683. E-mail: wyss.c@zzmk.unizh.ch.

$\uparrow$ This paper is dedicated to B. Guggenheim by his collaborators on the occasion of his 60 th birthday.
$16 S$ rRNA sequencing and phylogenetic analysis. DNA isolation, PCR amplification, sequencing, and phylogenetic analysis of $16 \mathrm{~S}$ ribosomal genes were performed by using a slight modification of a previously described procedure (7). Briefly, 16S rRNA was amplified by using one biotinylated primer and one normal primer. Biotinylated strands were separated by using streptavidin-coated paramagnetic beads (Dynabeads; Dynal, Hamburg, Germany) and were sequenced by using a Thermo Sequenase fluorescence-labelled primer cycle sequencing kit (Amersham, Braunschweig, Germany) and a model 4000 LI-COR automated sequencer (MWG-Biotech, Ebersberg, Germany) according to the manufacturer's instructions.

Nucleotide sequence accession numbers. The nearly complete $16 \mathrm{~S}$ ribosomal DNA sequence of type strain HA2P has been deposited in the EMBL, GenBank, and DDBJ nucleotide sequence databases under accession no. Y09959. The accession numbers of other sequences used for the phylogenetic analysis have been published previously (7).

\section{RESULTS AND DISCUSSION}

Cultural analysis of subgingival plaque microbiota is complicated by the physiological heterogeneity of the large number of fastidious bacterial species present, as well as by the complex network of synergistic and antagonistic metabolic interactions among all of the members of the population. Additional problems for isolation of pure strains are posed by the tightly aggregated forms characteristic of plaque and by the high motility of many oral bacteria. Recently, we developed the following two new tools to overcome some of these problems: (i) a new culture medium, medium OMIZ-Pat, was designed to be rich in nutrients and poor in growth inhibitors (7); and (ii) a limit dilution culture method for subgingival plaque samples was developed in order to immediately shield organisms from metabolic interactions with other members of the original population, to save slow growers from becoming overgrown, and to prevent invasion by motile cells $(6,7)$.

In conjunction with appropriate antibiotics, these tools allow routine isolation of oral spirochetes, including members of previously uncultured species (7). However, most oral Treponema genotypes identified in subgingival plaque by $16 \mathrm{~S}$ rRNA sequencing (1) remained uncultured.

Serum exsudate is a dominant component of the natural habitat of oral treponemes and probably contributes to the nutrition of all plaque bacteria. Indeed, serum has been used successfully for long-term cultivation of consortia of oral bacteria, including treponemes $(3,4)$, and has been considered an 


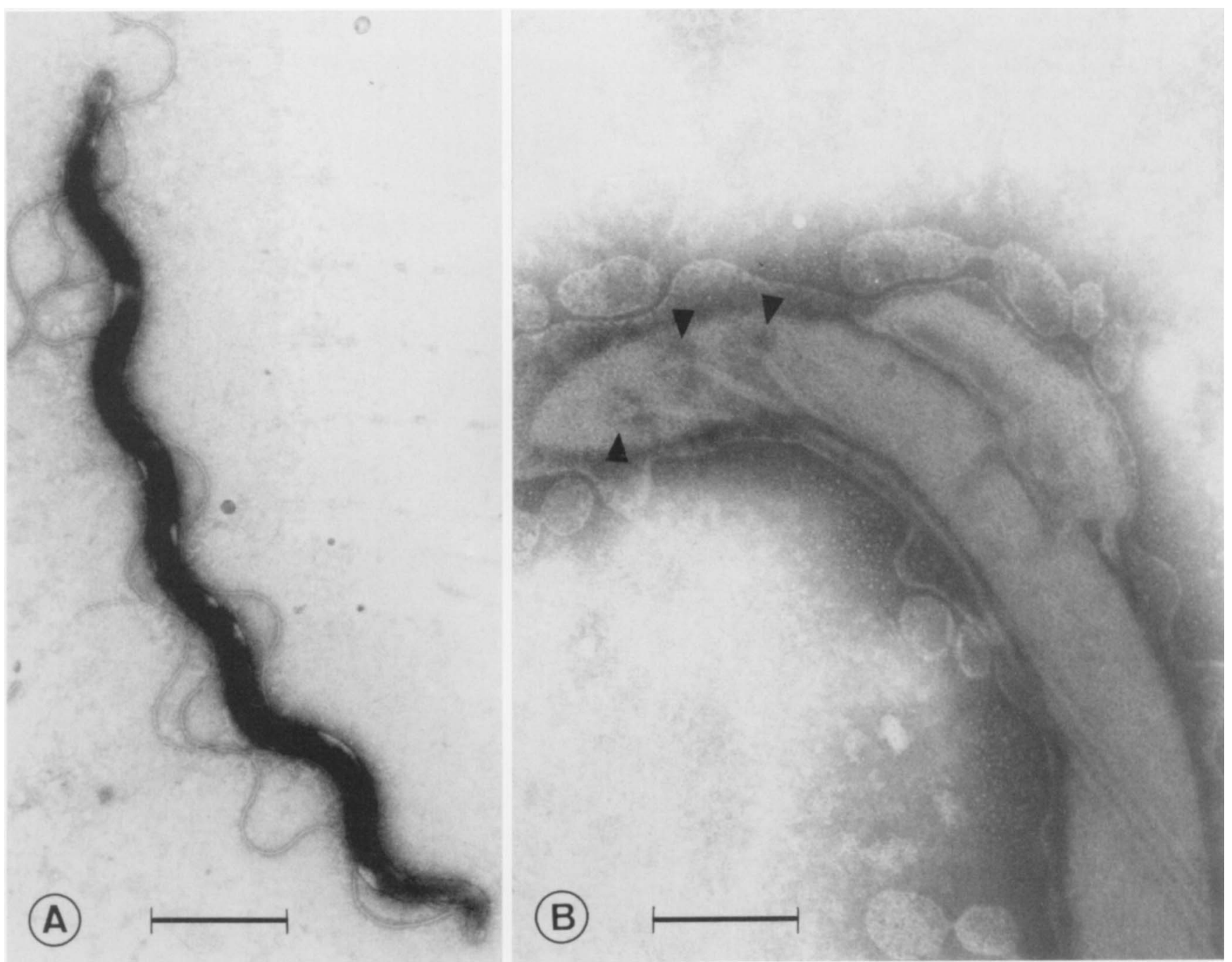

FIG. 1. (A) Transmission electron micrograph of a negatively stained strain $\mathrm{HA} 2 \mathrm{P}^{\mathrm{T}}$ cell. Bar $=1 \mu \mathrm{m}$. (B) Transmission electron micrograph of a negatively stained strain $\mathrm{HA} 2 \mathrm{P}^{\mathrm{T}}$ cell. The arrowheads indicate flagellar base plates. Bar $=0.2 \mu \mathrm{m}$.

important medium supplement for cultivation of oral treponemes. Intriguingly, however, we found that adding sera to media OMIZ-W1 and OMIZ-Pat inhibited the growth of all isolates of $T$. socranskii and $T$. maltophilum tested $(5,7)$. This inhibitory effect was seen with (heat-inactivated) fetal bovine serum (FBS), dialyzed FBS, horse serum, and local batches of human serum at concentrations as low as $0.5 \%$; yet, in contrast to $T$. socranskii and T. maltophilum, all strains of $T$. denticola and $T$. vincentii showed enhanced growth with serum supplements at concentrations as high as $10 \%$. Improved recovery of oral treponemes in medium OMIZ-Pat might therefore be expected if a serum devoid of inhibitory activity could be selected as the supplement.

By screening various commercial products in semiquantitative growth assays as described previously (7), we found that all four batches of Sigma human serum from male clotted blood tested (catalog no. H1388) stimulated growth of $T$. denticola ATCC $35405^{\mathrm{T}}$ and CD-1, T. maltophilum ATCC $51939^{\mathrm{T}}$, ATCC 51940, and ATCC 51941, T. socranskii subsp. socranskii ATCC $35534^{\mathrm{T}}$, and $T$. vincentii ATCC 35580 at concentrations ranging from 0.5 to $2 \%$. Higher concentrations of this serum inhibited growth of $T$. socranskii and T. maltophilum, whereas
$T$. denticola and $T$. vincentii were further stimulated up to the highest concentration tested $(10 \%)$. Similar activity was found with one batch of charcoal-treated human serum (catalog no. H4897; Sigma). In contrast, one batch of human serum from male AB plasma (catalog no. 2520; Sigma) and four locally produced sera from different individuals were inhibitory at a concentration of $0.5 \%$. All batches of FBS tested at a concentration of $1 \%$ inhibited the growth of $T$. socranskii and $T$. maltophilum. No information is presently available on the nature of either the growth-promoting components or the growth-inhibiting components in sera, except that both are retained by membranes with a nominal pore size that excludes molecules larger than ca. 10,000 Da.

Based on these results, medium OMIZ-Pat supplemented with $1 \%$ human serum (OMIZ-Pat/HuS) was employed in attempts to isolate novel spirochetes from human subgingival plaque. A sample was obtained from a $6-\mathrm{mm}$ pocket of a 54-year-old male patient with recurrent, treatment-resistant periodontitis; as determined by dark-field microscopy, this sample contained $18 \%$ motile rods, $58 \%$ small spirochetes, $4 \%$ intermediate spirochetes, and no large spirochetes. When the sample was cultured in OMIZ-Pat/HuS without antibiotics by 
the limit dilution technique, a viable count of $4 \times 10^{6}$ cells $/ \mathrm{ml}$ was obtained, and one of eight isolates was a spirochete $(T$. maltophilum). On this basis it appears that spirochetes represented $12 \%$ of the subgingival plaque microbiota growing in OMIZ-Pat/HuS. When the sample was cultured on Columbia blood agar plates, however, the viable count was $1.2 \times 10^{7}$ cells $/ \mathrm{ml}$, and $63 \%$ of all of the colonies were colonies of blackpigmenting bacteria. A comparison of the results of these three different analyses confirmed that a full description of plaque population structure is beyond the technical capacities of most laboratories. Since our current efforts are aimed at the isolation of spirochetes from plaque, selective culture conditions were also employed. When higher concentrations of the same plaque sample were cultured in OMIZ-Pat/HuS supplemented with $1 \mathrm{mg}$ of rifampin per liter and $100 \mathrm{mg}$ of fosfomycin per liter, growth of spirochetes was obtained in another seven wells. An analysis of all eight populations on streak plates containing solid OMIZ-Pat/HuS without antibiotics showed that they contained only spirochetes. However, one of the populations proved to be a mixture of $T$. denticola and another, small, as-yet-unidentified spirochete, raising the number of isolates to nine. Eight of these isolates represented small spirochetes and were identified as $T$. denticola (one isolate), $T$. maltophilum (five isolates), and a hitherto uncultered species (two isolates). One isolate, HA2P ${ }^{\mathrm{T}}$, however, was of intermediate size, and its description as the type strain of a novel species of oral treponemes is the subject of this report.

The morphology and SDS-PAGE protein pattern of isolate $\mathrm{HA} 2 \mathrm{P}^{\mathrm{T}}$ are shown in Fig. 1 and 2, respectively. Enzymatic activities and growth characteristics of this organism are summarized below in the description of the new species. The fact that $\alpha$-glucosidase activity was detected in strain HA2P ${ }^{\mathrm{T}}$ when cells were grown on maltose, starch, or glycogen but not when cells were grown on glucose may be the first evidence that environmental regulation of metabolic activities occurs in oral treponemes.

A phylogenetic analysis (Fig. 3) based on a comparison of about 1,350 bp (positions 54 to 1388 in Escherichia coli $16 \mathrm{~S}$ rRNA) revealed that the $16 \mathrm{~S}$ rRNA sequence of strain HA2P was distinct from the $16 \mathrm{~S}$ rRNA sequences of all other cultured spirochetes and showed $88 \%$ similarity to the sequence of its closest cultured relative. However, a high level of similarity $(98 \%)$ was found when the HA2P ${ }^{\mathrm{T}}$ 16S rRNA sequence was compared with the $16 \mathrm{~S}$ rRNA sequence of $16 \mathrm{~S}$ rRNA clone NZM399, a phylotype belonging to treponeme cluster 19 (1). Successful isolation of strain HA2P $\mathrm{P}^{\mathrm{T}}$ and classification of this organism as a cluster 19 oral treponeme underline the validity of our molecular genetic data on the diversity of oral treponemes (1) and of our efforts in medium development $(5,7)$. However, to fully describe the physiological potential of cluster 19 treponemes, it will be necessary to obtain additional isolates. As present culture techniques do not allow conclusive determination of the distribution of these novel organisms in different plaque samples, we have begun to study the epidemiology of these treponemes by culture-independent hybridization techniques.

Description of Treponema amylovorum sp. nov. Treponema amylovorum (a.my.lo.vo' rum. Gr. n. amylum, starch; L. V. voro, to devour; M. L. adj. amylovorum, starch devouring) is an intermediate-size, obligately anaerobic, helically coiled, motile treponeme. Strain HA2 $\mathrm{P}^{\mathrm{T}}$, which was isolated from subgingival plaque of a deep human periodontal lesion, is designated the type strain and has been deposited in the American Type Culture Collection (Rockville, Md.) under accession number ATCC 700288.

As shown in Fig. 1, the cells are ca. $0.25 \mu \mathrm{m}$ in diameter and

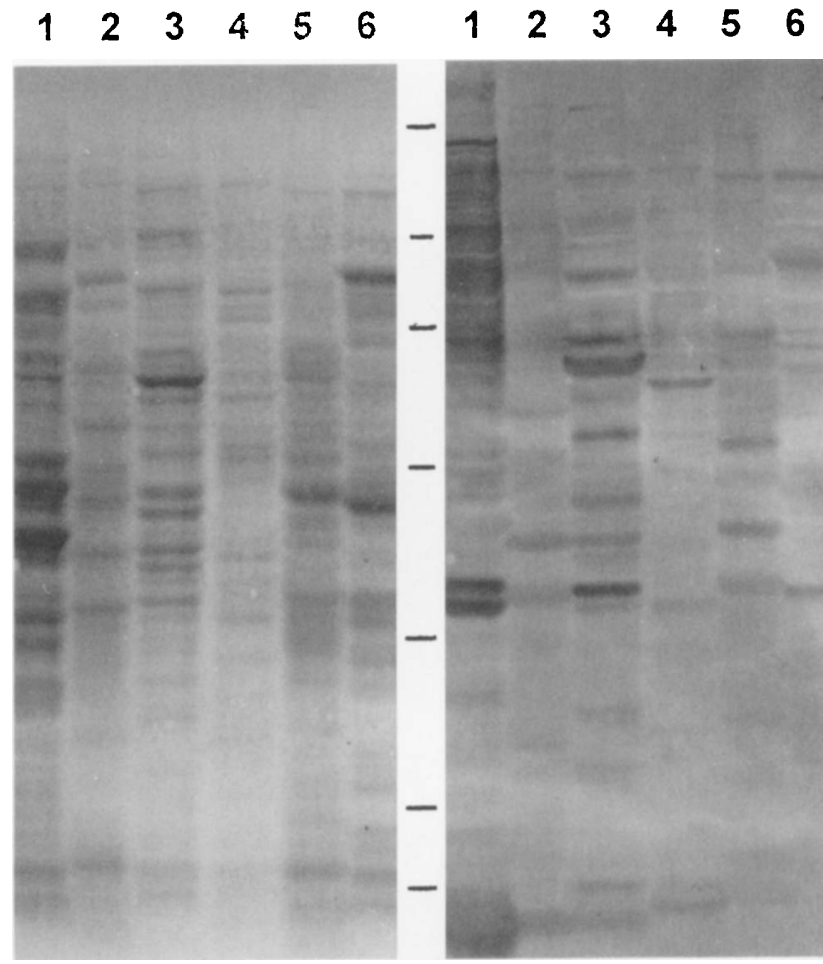

A

B

FIG. 2. Protein and antigen profiles of representative strains of cultivable oral spirochetes after SDS-PAGE on a 7.5 to $15 \%$ polyacrylamide gradient and electroblotting onto nitrocellulose. Lane 1 , novel isolate $\mathrm{HA}_{2} \mathrm{P}^{\mathrm{T}}$; lane $2, T$. vincentii LA-1 (= ATCC 35580); lane 3, T. maltophilum ATCC 51939 ${ }^{\mathrm{T}}$; lane 4, T. denticola ATCC $35405^{\mathrm{T}}$; lane 5 , T. socranskii subsp. socranskii ATCC $35536^{\mathrm{T}}$; lane $6, T$. pectinovorum ATCC $33768^{\mathrm{T}}$. (A) Blot stained for protein with copper phthalocyaninetetrasulfonic acid. (B) Same blot as in panel A after removal of protein stain and immunostaining with serum from a periodontitis patient. The positions of molecular weight markers (molecular weights [from top to bottom], 200,000, 97,000, 69,000, $46,000,30,000,21,000$ and 13,000 ) are indicated between the panels.

approximately $7 \mu \mathrm{m}$ long, with a wavelength of ca. $1.2 \mu \mathrm{m}$ and an amplitude of ca. $0.3 \mu \mathrm{m}$. They have six periplasmic flagella; three flagella originate at each cell end, and the flagella overlap in the central region of the cell (i.e., flagellation type 3:6:3). In liquid media of low viscosity cells exhibit active cellular rotation and jerky flexing but no directional motility. Translational movement, however, is seen in media of higher viscosity or when cells creep along a surface. Cells can be stored at temperatures below $-70^{\circ} \mathrm{C}$ in medium supplemented with 10 to $20 \%$ glycerol.

(i) Colonies. When streaked onto OMIZ-Pat/HuS agarose plates, T. amylovorum forms dense, off-white, subsurface colonies up to $3 \mathrm{~mm}$ in diameter within 5 days of inoculation.

(ii) Cultural characteristics. T. amylovorum does not grow in chemically defined OMIZ-W1 medium and requires the addition of yeast extract and/or Neopeptone (or fractions thereof). Addition of $1 \%$ human serum is highly stimulatory, whereas FBS or higher concentrations of human serum are inhibitory. Growth of strain HA2P $\mathrm{P}^{\mathrm{T}}$ is accompanied by acid production, as judged by phenol red indicator in the medium, and strictly depends on the presence of at least one of the following carbohydrates: glucose, maltose, starch, or glycogen. Acid is produced from these carbohydrates. None of the other carbohydrates tested (at a concentration of $2 \mathrm{~g} /$ liter) support growth; these carbohydrates include D-arabinose, D-cellobiose, 
Distance 0.1

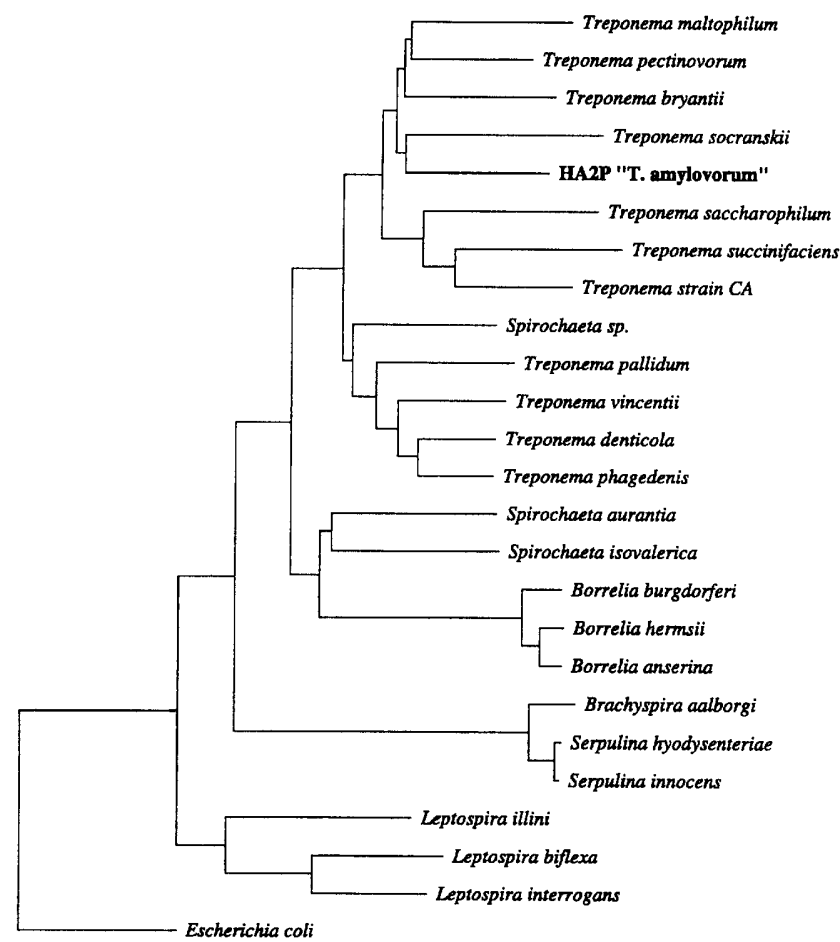

FIG. 3. Phylogenetic tree showing the relationship of novel strain $H A 2 \mathbf{P}^{\mathrm{T}}$ to other spirochetes based on a comparison of $16 \mathrm{~S}$ rRNA sequences, with E. coli as the outgroup. Scale bar $=10 \%$ difference in nucleotide sequences as determined by measuring the lengths of the horizontal lines connecting two sequences.

D-fructose, D-fucose, D-galactose, D-galacturonic acid, D-glucuronic acid, D-lactose, D-mannitol, D-mannose, D-melibiose, Dribose, $\mathrm{D}$-sucrose, D-trehalose, D-xylose, L-arabinose, L-fucose, L-rhamnose, L-sorbose, and L-xylose. Following the exponential growth phase, with an estimated doubling time of less than $4 \mathrm{~h}$, cells of $\mathrm{HA} 2 \mathrm{P}^{\mathrm{T}}$ rapidly loose viability and disintegrate into small vesicles. Catalase activity is not observed. With API ZYM strips the only (weak) enzyme activities detected in cells grown on glucose are alkaline and acid phosphatases, naphtholphosphohydrolase, $\mathrm{C} 4$ esterase, and $\alpha$-fucosidase. In cells grown on either maltose, starch, or glycogen weak $\alpha$-glucosidase activity is also detected. Strain $\mathrm{HA}_{2} \mathrm{P}^{\mathrm{T}}$ is resistant to $1 \mathrm{mg}$ of rifampin per liter and $100 \mathrm{mg}$ of fosfomycin per liter.

(iii) Distinguishing characteristics. On the basis of a phylogenetic comparison of $16 \mathrm{~S}$ rRNA sequences, $T$. amylovorum is a species that is genetically distinct from previously described treponemes. Its SDS-PAGE protein and antigen profiles and its Western blot profile are readily distinguished from those of the other five cultivable, oral Treponema species (Fig. 2). Furthermore, size and flagellation, as well as rapid flexing motility, clearly distinguish $T$. amylovorum from other treponeme species, such as T. denticola, $T$. maltophilum, T. pectinovorum, and $T$. socranskii. In contrast to the two asaccharolytic oral species, $T$. denticola and $T$. vincentii, growth of strain $\mathrm{HA} 2 \mathrm{P}^{\mathrm{T}}$ is strictly carbohydrate dependent, and the range of carbohydrates utilized is clearly distinct from the range of carbohydrates utilized by $T$. maltophilum, T. pectinovorum, and T. socranskii.

\section{ACKNOWLEDGMENTS}

We thank C. Weiss, V. Zängerle, and M. Kachler for expert technical assistance and S. Shapiro for a critical review of the manuscript.

This work was supported in part by grant $01 \mathrm{KI} 9318$ from the Deutsches Bundesministerium für Bildung und Forschung.

\section{REFERENCES}

1. Choi, B. K., B. J. Paster, F. E. Dewhirst, and U. B. Göbel. 1994. Diversity of cultivable and uncultivable oral spirochetes from a patient with severe destructive periodontitis. Infect. Immun. 62:1889-1895.

2. Haffajee, A. D., and S. S. Socransky. 1994. Microbial etiological agents of destructive periodontal diseases. Periodontology 2000 5:78-111.

3. ter Steeg, P. F., and J. S. van der Hoeven. 1990. Growth stimulation of Treponema denticola by periodontal microorganisms. Antonie van Leeuwenhoek 57:63-70.

4. ter Steeg, P. F., J. S. van der Hoeven, M. H. de Jong, P. J. J. van Munster, and M. J. H. Jansen. 1987. Enrichment of subgingival microflora on human serum leading to accumulation of Bacteroides species, peptostreptococci, and fusobacteria. Antonie van Leeuwenhoek 53:261-271.

5. Wyss, C. 1992 . Growth of Porphyromonas gingivalis, Treponema denticola, $T$. pectinovorum, $T$. socranskii, and $T$. vincentii in a chemically defined medium. J. Clin. Microbiol. 30:2225-2229.

6. Wyss, C. 1995. Sticky, a novel phenotype of Campylobacter rectus. Microb. Ecol. Health Dis. 8:175-179.

7. Wyss, C., B. K. Choi, P. Schüpbach, B. Guggenheim, and U. B. Göbel. 1996. Treponema maltophilum sp. nov., a small oral spirochete isolated from human periodontal lesions. Int. J. Syst. Bacteriol. 46:745-752. 